establish their diagnostic value and value in management of abnormal skull shape.

Methods Retrospective chart review conducted as a 2 part study in children who were referred with a skull $\mathrm{x}$-ray.

Part A: Referrals of abnormal skull shapes to the National Pediatric Craniofacial Center (NPCC), Temple Street Children's Hospital, between 1st January 2015 and 30th May 2017

Part B: Children who underwent surgery for a confirmed craniosynostosis between 1st January 2011 - 25th October 2017.

Results and Findings Part A: 300 children were referred with 59 skull x-rays. This represented $20 \%$ of all patients referred during the time period. Of these $44(75 \%)$ were found to be a match with 15 (25\%) not matching the final clinical diagnosis.

Part B: 274 children underwent surgery for a confirmed craniosynostosis between 1st January 2011 - 25th October 2017. 63 pts had skull x-rays on referral- this represents $23 \%$ of all operated children in the time period. Of these 41 $(63 \%)$ were found to be a match with 17 (29\%) not matching the final clinical diagnosis. $5(8 \%)$ were inconclusive.

Conclusions Part A: In 25\% of the children referred to the NPCC with abnormal skull shape, their clinical diagnosis did not match their $\mathrm{x}$-ray report. As such, skull $\mathrm{x}$-rays did not contribute to their management.

Part B: In 35\% of children who underwent surgery for craniosynostosis, their clinical diagnosis did not match their radiological diagnosis.

In the remaining children who had a skull x-ray performed and underwent surgery for craniosynostosis, the majority $(88 \%)$ had a subtype of craniosynostosis which our clinical team who feel confident to diagnosis clinically without imaging.

As such, it can be said that in $92 \%$ of children who underwent surgery at the NPCC the x-ray did not contribute to their management.

We recommend clinicians should check with the NPCC with respect to the protocol for $\mathrm{x}$-rays where craniosynostosis is suspected as in most cases the diagnosis is clinically obvious to the craniofacial surgeon.

\section{GP10 CHRONIC NONBACTERIAL OSTEOMYELITIS; THE IRISH EXPERIENCE}

1,2,3 Daire O'Leary*, ${ }^{4}$ Clodagh Lowry, ${ }^{3}$ Emma MacDermott, ${ }^{5}$ Gerry Wilson, 3,5 Orla Killeen. ${ }^{1}$ School of Medicine, UCD, Dublin, Ireland; ${ }^{2} N C R C$, Dublin, Ireland; ${ }^{3} \mathrm{OLCHC}$, Dublin, Ireland; ${ }^{4} \mathrm{CUH}$, Temple St, Dublin, Ireland; ${ }^{5}$ School of Medicine, UCD, Dublin, Ireland

\subsection{6/archdischild-2019-epa.77}

Background Chronic nonbacterial osteomyelitis (CNO) is a rare autoinflammatory disease affecting bone with an estimated prevalence of 1 in $10^{5}$. Untreated CNO can result in complications such as vertebral compression fractures and leg length discrepancy. Limited data on different clinical phenotypes and efficacy of treatments (such as NSAIDs, steroids, methotrexate, bisphosphonates and biologic agents) makes prognosis challenging.

Objectives To describe the clinical phenotype of an Irish cohort of patients with $\mathrm{CNO}$ including treatment response.

Methods Retrospective chart review of current patients attending the National Centre for Paediatric Rheumatology and the
Rheumatology Department in the Children's University Hospital. Clinical charts, radiology and histology reports were reviewed. All data was coded and statistical analysis was performed in $\mathrm{R}$.

Results Clinical charts of 37 patients with $\mathrm{CNO}$ were reviewed. The median age at onset was 8.5 years, median follow-up was 3 years and the median number of sites 3 (1-21). F:M was 2.7:1. 94\% had multifocal disease. $35 \%$ had a personal history of an associated disease such as psoriasis, inflammatory arthritis or inflammatory bowel disease. A first- or second-degree family history of an associated disease was present in 54\%. All patients underwent whole-body MRI prior to diagnosis. All patients with unifocal disease underwent biopsy to outrule infection or malignancy.

Treatment escalation beyond NSAIDs was required in 51\% with biologic agents being used in $84 \%$ of those requiring second-line treatment. The indications for second-line treatment were in keeping with recent CARRA guidelines; persistent active disease on NSAIDS $(n=12)$, the presence of spinal lesions $(n=5)$, the presence of a physeal lesion $(n=1)$ and preexisting JIA $(n=1)$. Biologic agents led to symptomatic improvement in all patients while none of the patients in this cohort responded to bisphosphonates; response to methotrexate was variable.

Conclusions There was a low incidence of unifocal disease and a high incidence of either a personal or family history of associated diseases in this cohort. Those with unifocal disease were less likely to have an associated inflammatory disease or to require second-line treatment. The rate of progression to biologic treatment was higher than that reported most other cohorts. Multinational collaborative consistent data collection with clearly defined outcomes is required to ascertain the association between different $\mathrm{CNO}$ phenotypes and outcomes.

\section{GP11 EFFICACY OF SOFT TISSUE SURGERY FOR FLEXION KNEE CONTRACTURES IN CHILDREN WITH CEREBRAL PALSY}

Maria Volkova*, Konstantin Zherdev, Oleg Chelpachenko, Ivan Nikitenko. National Medical Research Center for Children's Health Federal state autonomous institution of the Russian Federation Ministry of Health, Moscow, Russian Federation

\subsection{6/archdischild-2019-epa.78}

Aim of the Study To assess the results of concomitant hamstring lengthening and plication of the patellar tendon.

Methods Retrospective data of 38 patients (72 knees), 27 boys (71\%) and 11 girls (29\%), with flexion knee contractures due to cerebral palsy who were treated from 2012 to 2018 were reviewed. Average patient age was $9.8 \quad(\mathrm{SD}=2)$ years (range, 7 - 12 years). The degree of knee contracture, ambulatory status (using the Gillette Functional Assessment Questionnaire (Gillette FAQ) 10-point scale) were evaluated before surgery and after rehabilitation period. The follow-up period ranged from 6 months to 6 years. 24 Patients (42 knees), 11 boys (46\%), 13 girls (54\%) with a diagnosis of one lower limb shortening due to hemihypoplasia, LeggCalve-Perthes disease or tumor mass in metaphysis, without a diagnosis of cerebral palsy, were assigned to reference group to determining normal range of knee extension in children 7-12 years old. Average patient age in this group was 10.6 $(S D=2$, range, $7-12)$ years. In the routine practice, all children of the reference group underwent CT-scan of the lower 
extremities, from which the angle of knee joint extension was determined. Statistics were given as mean $(\mathrm{SD}=$ standart deviation). A paired t test was used to detect differences between scores. Statistical significance was determined as a pvalue $<0,05$.

Main results Preoperative knee flexion deficiency ranged from 10 to $90^{\circ}$. Mean preoperative flexion contracture was $26.6^{\circ}$ $(\mathrm{SD}=18.9)$. After surgery knee flexion contracture $\left(6.9^{\circ}\right.$, $\mathrm{SD}=10.4)$ has significantly decreased $(\mathrm{p}<0,05)$. Mean correction amount of flexion contracture was $77.7 \%(\mathrm{SD}=22.3 \%)$. Mean preoperative score of Gillette FAQ scale was $2(\mathrm{SD}=$ 1). After surgery mean Gillette FAQ score $(3, \mathrm{SD}=1)$ has significantly improved $(p<0,05)$. Three patients had following complications: synovitis knee joints after immobilization period, $20^{\circ}$ and $60^{\circ}$ knee joints extensor contractures at examination after one year since surgery. For patients of the reference group, the average angle of knee joint extension was $1 \circ$ recurvation $(\mathrm{SD}=3)$.

Conclusions The obtained data indicate a rather high efficacy of surgical treatment both in terms of goniometric and functional indicators. However, the average angle of knee joint extension after surgery is significantly less than the reference value $(p<0,05)$ and it is necessary to consider possible complications associated with requirement for long-term postsurgery immobilization.

\section{GP12 DEVELOPMENTAL DYSPLASIA OF HIP - SCREENING WITHOUT RADIOGRAPHS; IS IT TIME?}

1,2Elinor Jenkins*, ${ }^{1}$ Alison Bell, ' Jane Cassidy, ${ }^{1}$ Evangeline Morris, ${ }^{1}$ Christopher 0 Loughlin, ${ }^{2}$ John Kelleher, ${ }^{2}$ Paula Kelly. ${ }^{1}$ Trinity College School of Medicine, Dublin, Ireland; ${ }^{2}$ The Coombe Women and Infants University Hospital, Dublin, Ireland

\subsection{6/archdischild-2019-epa.79}

Background The optimum screening programme for developmental dysplasia of the hip remains elusive. Ireland has introduced a new national screening guideline based on selective USS.

Aim This study aims to determine the sensitivity of the new national guideline through a retrospective audit in one large teaching hospital.

Methods All infants referred for DDH imaging between 08/ $09 / 18$ and $08 / 03 / 19$ were included in the study. Infants with a normal USS (table 1) at 6 weeks were followed to radiograph at 6 months. A chart review of all infants with normal USS but abnormal or concerning radiograph was conducted to determine outcome.

\begin{tabular}{|c|c|c|c|}
\hline & DDH & No DDH & \\
\hline Hip USS + ve & $\begin{array}{l}\text { True Positive } \\
\mathrm{N}=61\end{array}$ & $\begin{array}{l}\text { Presume } \mathrm{N}=0 \text { as } \\
\text { charts not reviewed }\end{array}$ & PPV - not calculated \\
\hline Hip USS - ve & $\begin{array}{l}\text { False Negative } \\
\mathrm{N}=61 \\
\text { Sensitivity }=\mathrm{TP} / \mathrm{TP}+\mathrm{FN} \\
\text { Sensitivity }=50 \%\end{array}$ & $\begin{array}{l}\text { True Negative } \\
\mathrm{N}=477\end{array}$ & $\begin{array}{l}\mathrm{NPV}=\mathrm{TN} / \mathrm{FN}+\mathrm{TN} \\
\mathrm{NPV}=\mathbf{8 8 . 6 \%}\end{array}$ \\
\hline
\end{tabular}

Results 716 infants (17.3\% of births) were referred for imaging based on clinical exam and current risk criteria. Of these,
655 infants (91.5\%) had normal USS hips at 6 weeks. At 6 months 158 infants (24\%) with normal USS had an abnormal or concerning $\mathrm{x}$-ray pelvis. $131(11.6 \%)$ were subject to surveillance but later discharged. $61(9.3 \%)$ remain under surveillance, 13 (2\%) required bracing. 74 (11\%) DNA'd follow up appointments. To date none of the infants who had a normal USS have required surgery.

Discussion and conclusion A retrospective audit using USS with the addition of radiograph identified a potential 0 9.3\% false negative rate and a sensitivity of $50 \%$. While these figures are concerning, following the 61 infants currently under surveillance is important. Should no infant with normal USS require surgery clinicians may be more confident in the new national guideline.

\section{GP13 DIFFERENTIAL MIRNA EXPRESSION IN CF AND GENE CORRECTED IPSC-DERIVED LUNG ORGANOIDS}

${ }^{1}$ Irene Oglesby*, ${ }^{2}$ BMcCauley Katherine," ${ }^{2}$ Anjali Jacob, ${ }^{2}$ NKotton Darrell, ${ }^{2}$ Finn Hawkins, ${ }^{1}$ Killian Hurley. ${ }^{1} R C S I, E R C$, Dept. of Respiratory Medicine, Beaumont, Ireland; ${ }^{2}$ Center for Regenerative Medicine, Boston University and Boston Medical Center, Boston, USA

\subsection{6/archdischild-2019-epa.80}

Background Cystic fibrosis (CF) is one of the most common lethal genetic diseases and is caused by mutations in the CFTR gene where a deletion of phenylalanine at position 508 (F508del) accounts for the most prevalent mutation worldwide. MicroRNA (miRNA) are small non-coding RNAs that can regulate up to two thirds of gene expression in human cells and have been shown to be dysregulated in the CF airway and implicated in the direct control of CFTR Induced Pluripotent stem cells (iPSC) can be routinely generated from any patient and contain that person's unique genetic background, CF mutation and can form all the cell types affected by $\mathrm{CF}$ representing an attractive tool to advance the understanding of CF. Recent advances in iPSC technology have led to an in vitro platform of airway epithelial organoids derived from CF and CF-corrected cells for modelling CF lung disease however this model requires further molecular characterisation. We hypothesise that specific miRNA play a key role in the pathogenesis of CF lung disease and airway organoids generated from patient derived iPSCs can better elucidate this role.

Methods Cystic fibrosis patient specific iPSC derived airway organoids were generated from an individual homozygous for the F508del mutation $(\Delta \mathrm{F} 508 / \Delta \mathrm{F} 508)$ and its gene corrected counterpart ( $\Delta \mathrm{F} 508 /$ wild-type [WT]) using established protocols. Expression of a miRNA panel we have previously shown to regulate CFTR (miR-145, miR-223 and miR-494) were examined in CF versus CF corrected iPSC lines at Day 0 and Day 32 following directed differentiation of iPSC into proximal airway organoids.

Results In preliminary work we have observed that miRNA known to target CFTR are detectable in CF and gene-corrected iPSC lines and differential expression is observed between cells at the pluripotent stage (iPSC) at Day 0 and cells that have been differentiated in $3 \mathrm{D}$ as organoids to airway-like cells (bronchospheres) at Day 32. Importantly, there is differential expression of key CFTR related miRNA when we compare CF patient-derived bronchospheres to those where the F508del mutation has been corrected. Increased expression of miR-494 was observed in CF vs. CF corrected 